\title{
Partial radiogenic heat model for Earth revealed by geoneutrino measurements
}

\author{
The KamLAND Collaboration*
}

The Earth has cooled since its formation, yet the decay of radiogenic isotopes, and in particular uranium, thorium and potassium, in the planet's interior provides a continuing heat source. The current total heat flux from the Earth to space is 44.2 $\pm 1.0 \mathrm{TW}$, but the relative contributions from residual primordial heat and radiogenic decay remain uncertain. However, radiogenic decay can be estimated from the flux of geoneutrinos, electrically neutral particles that are emitted during radioactive decay and can pass through the Earth virtually unaffected. Here we combine precise measurements of the geoneutrino flux from the Kamioka Liquid-Scintillator Antineutrino Detector, Japan, with existing measurements from the Borexino detector, Italy. We find that decay of uranium-238 and thorium-232 together contribute 20.0 flux. The neutrinos emitted from the decay of potassium-40 are below the limits of detection in our experiments, but are known to contribute 4 TW. Taken together, our observations indicate that heat from radioactive decay contributes about half of Earth's total heat flux. We therefore conclude that Earth's primordial heat supply has not yet been exhausted.

T he Kamioka Liquid-Scintillator Antineutrino Detector (KamLAND) Collaboration reported the results of the first study of electron antineutrinos $\left(\bar{v}_{\mathrm{e}} \mathrm{s}\right)$ produced within the Earth in 2005 (ref. 1). The KamLAND data indicated an excess of the $\bar{\nu}_{\mathrm{e}}$ flux at energies in the range consistent with the decay chains of ${ }^{238} \mathrm{U}$ and ${ }^{232} \mathrm{Th}$. The rate was consistent with geophysical expectations for heavy-element concentrations throughout the Earth's interior. The statistical significance improved as more data were obtained ${ }^{2}$. Recently, the Borexino Collaboration at Gran Sasso reported an excess of events they attribute to geoneutrinos ${ }^{3}$. While Borexino convincingly confirms the KamLAND excess, neither result is precise enough to provide much guidance for geophysical models. In this paper we present more recent KamLAND results that begin to constrain the models.

The present analysis is possible because of the recently improved sensitivity to geoneutrinos in the KamLAND experiment. The KamLAND geoneutrino background is dominated by $\bar{\nu}_{\mathrm{e}} \mathrm{s}$ from commercial nuclear reactors and the ${ }^{13} \mathrm{C}(\alpha, n){ }^{16} \mathrm{O}$ reaction initiated by the decay of radioactive contaminants in the detector. In recent years the reactor $\bar{v}_{\mathrm{e}}$ flux, which is outside the control of the experiment, was significantly reduced ${ }^{2}$ because of an extended shutdown of the Kashiwazaki-Kariwa nuclear power station following an earthquake in July 2007. In the meantime, at great effort, the purity of the KamLAND scintillator was improved, eliminating most of the ${ }^{210} \mathrm{~Pb}$ that feeds the decay chain responsible for the production of $\alpha$-particles from ${ }^{210}$ Po decay. The background from the ${ }^{13} \mathrm{C}(\alpha, n){ }^{16} \mathrm{O}$ reaction went down by a factor of $\sim 20$.

The measurement presented here is based on data collected between 9 March 2002 and 4 November 2009, and includes the data used in our previous publications ${ }^{1,2}$. The total exposure to $\bar{\nu}_{\mathrm{e}}$ is $(3.49 \pm 0.07) \times 10^{32}$ target proton years, a fivefold increase from the first KamLAND report ${ }^{1}$. The expected signal in one geophysical model $^{4}$ increases from 19 events to 106 events.

\section{Earth composition model}

Analyses of seismic waves indicate a shell structure for the Earth's interior, conventionally denoted as crust, upper mantle, lower mantle, outer core and inner core. Although the mechanical properties and bulk composition of the shells are well established, their detailed composition, including the abundances of radiogenic species, remains uncertain.

Since the model assumptions are not well grounded, the predictions have large uncertainties. The bulk silicate Earth (BSE) model of ref. 5, for example, assumes that the primordial Earth was formed from accretion of matter from the same nebula that formed the sun and the rest of the planets, and that the BSE abundances of refractory lithophile elements such as $U$ and Th can be determined by a combination of measured elemental abundances of chondritic meteorites and mantle peridotites. The composition deduced from this model results in a radiogenic heat production of $8 \mathrm{TW}$ from the ${ }^{238} \mathrm{U}$ decay chain, $8 \mathrm{TW}$ from the ${ }^{232} \mathrm{Th}$ decay chain and $4 \mathrm{TW}$ from ${ }^{40} \mathrm{~K}$ (ref. 6). In this model, the radiogenic heat contribution is nearly half of the Earth's total heat flow $(44.2 \pm 1.0$ TW, ref. 7; see also Supplementary Note S1). Clearly, quantitative information about the radiogenic, heat-producing elements is essential for establishing the energy budget, which in turn is key to understanding the Earth's formation and evolution.

\section{Geoneutrino flux}

The Earth is nearly transparent to neutrinos because they interact only through the weak force. Geoneutrinos are a unique, direct window into the interior of the planet. Accurately mapping neutrino sources inside the Earth by measuring geoneutrino fluxes at the surface is an inverse problem requiring multiple detection sites. The prospects for multisite measurements have been discussed in refs 4,8, and an analysis of the likely sensitivity of such measurements was discussed in ref. 9. For the present work, which has limited statistics, a simple BSE-inspired reference model $^{4}$ for the radiogenic material distribution is employed. The model assumes that $\mathrm{U}$ or $\mathrm{Th}$ is present in the crust and mantle but not in the core. The effects of radiogenic material in the local geology of Kamioka are carefully assessed but account for less than $10 \%$ of the total expected flux. 
The differential geoneutrino flux at a position $\mathbf{r}$ is determined from the isotopic abundances $a_{i}\left(\mathbf{r}^{\prime}\right)$ at the location of the sources, $\mathbf{r}^{\prime}$,

$$
\frac{\mathrm{d} \Phi\left(E_{v}, \mathbf{r}\right)}{\mathrm{d} E_{v}}=\sum_{i}^{\text {isotopes }} A_{i} \frac{\mathrm{d} n_{i}\left(E_{v}\right)}{\mathrm{d} E_{v}} \int_{\oplus} \mathrm{d}^{3} \mathbf{r}^{\prime} \frac{a_{i}\left(\mathbf{r}^{\prime}\right) \rho\left(\mathbf{r}^{\prime}\right) P\left(E_{v},\left|\mathbf{r}-\mathbf{r}^{\prime}\right|\right)}{4 \pi\left|\mathbf{r}-\mathbf{r}^{\prime}\right|^{2}}
$$

where the integration extends over the Earth's volume, $A_{i}$ is the decay rate per unit mass, $\mathrm{d} n_{i}\left(E_{v}\right) / \mathrm{d} E_{v}$ is the $\bar{\nu}_{\mathrm{e}}$ energy spectrum for each mode of decay, $a_{i}\left(\mathbf{r}^{\prime}\right)$ is in units of isotope mass per unit rock mass, $\rho\left(\mathbf{r}^{\prime}\right)$ is the rock density and $P\left(E_{v},\left|\mathbf{r}-\mathbf{r}^{\prime}\right|\right)$ is the $\bar{v}_{\mathrm{e}}$ 'survival' probability due to the phenomenon of oscillation after travelling a distance $\left|\mathbf{r}-\mathbf{r}^{\prime}\right|$. For the present purpose, the $\bar{\nu}_{\mathrm{e}}$ survival probability is well approximated by the two-flavour oscillation formula,

$$
P\left(E_{v}, L\right) \simeq 1-\sin ^{2} 2 \theta_{12} \sin ^{2}\left(\frac{1.27 \Delta m_{21}^{2}\left[\mathrm{eV}^{2}\right] L[\mathrm{~m}]}{E_{v}[\mathrm{MeV}]}\right)
$$

where $L=\left|\mathbf{r}-\mathbf{r}^{\prime}\right|$. 'Matter effects' on neutrino oscillations ${ }^{10}$ are expected to change equation (2) by about $1 \%$, which is negligible compared with the statistical uncertainty. The oscillation parameters $\Delta m_{21}^{2}$ and $\sin ^{2} 2 \theta_{12}$ are determined with substantial accuracy by a combined statistical analysis with KamLAND's measurement of $\bar{\nu}_{\mathrm{e}} \mathrm{s}$ produced at nuclear reactors and data from solar-neutrino experiments (assuming charge-parity-time (CPT) symmetry ${ }^{10}$ ), and are given in the next section. Given the size of the Earth and the values of the neutrino oscillation parameters, for the energy range of detectable geoneutrinos the second sine function in equation (2) is well averaged over the volume of the Earth, giving $P\left(E_{v}, L\right) \simeq 1-0.5 \sin ^{2} 2 \theta_{12}$ to an excellent approximation.

\section{Geoneutrino detection}

KamLAND is located under Mount Ikenoyama $\left(36.42^{\circ} \mathrm{N}\right.$, $\left.137.31^{\circ} \mathrm{E}\right)$, near the town of Kamioka, Japan. The underground site provides an effective overburden of $2,700 \mathrm{~m}$ water equivalent, reducing the cosmic-ray-induced atmospheric muon flux to $5.37 \pm 0.41 \mathrm{~m}^{-2} \mathrm{~h}^{-1}$ (ref. 11). The $\bar{\nu}_{\mathrm{e}} \mathrm{s}$ are detected in $1 \mathrm{kt}$ of liquid scintillator (LS) through the inverse $\beta$-decay reaction, $\bar{v}_{\mathrm{e}}+p \rightarrow e^{+}+n$, with a $1.8 \mathrm{MeV}$ neutrino energy threshold. This threshold cuts off much of the geoneutrino signal from the ${ }^{238} \mathrm{U}$ and ${ }^{232} \mathrm{Th}$ decay chains and renders the detector insensitive to ${ }^{40} \mathrm{~K}$ (other unobserved isotopes such as ${ }^{235} \mathrm{U}$ contribute negligibly to the heating). Using the cross-section from ref. 12, the expected rate of geoneutrino events from the geological reference model ${ }^{4}$ is $3.80 \times 10^{-31} \bar{\nu}_{\text {e }}$ per target proton per year. $79 \%$ of this rate is due to ${ }^{238} \mathrm{U}$ decays. The prompt scintillation light from the $e^{+}$provides an estimate of the incident $\bar{\nu}_{\mathrm{e}}$ energy, $E_{\bar{\nu}_{\mathrm{e}}} \simeq E_{\mathrm{p}}+\bar{E}_{n}+0.8 \mathrm{MeV}$, where $E_{\mathrm{p}}$ is the sum of the positron's kinetic energy and its annihilation energy, and $\bar{E}_{n}$ is the average neutron recoil energy of $O(10 \mathrm{keV})$. The neutron is captured on a proton, emitting a $2.2 \mathrm{MeV} \gamma$-ray after a mean delay time of $207.5 \pm 2.8 \mu$ s following the positron's annihilation. The delayed-coincidence signal is a powerful tool for reducing backgrounds.

The data collected between 9 March 2002 and 4 November 2009 represents a total live-time of 2,135 days. The number of target protons in the spherical fiducial volume of radius $6.0 \mathrm{~m}$ is estimated to be $(5.98 \pm 0.12) \times 10^{31}$, resulting in a total exposure of $(3.49 \pm 0.07) \times 10^{32}$ target proton years. Data taken during the LS purification activities exhibited increased PMT noise and were excluded from the data set.

The fluxes of reactor $\bar{v}_{\mathrm{e}} \mathrm{s}$ are analysed together with the geoneutrinos and are calculated using instantaneous thermal power, burnup and refuelling records for all commercial reactors in Japan, as provided by a consortium of Japanese electric power companies. Only four fissile isotopes, ${ }^{235} \mathrm{U},{ }^{238} \mathrm{U},{ }^{239} \mathrm{Pu}$ and ${ }^{241} \mathrm{Pu}$, contribute significantly to the $\bar{\nu}_{\mathrm{e}}$ spectrum ${ }^{13-15}$. Spectral uncertainties were further constrained according to ref. 16 .
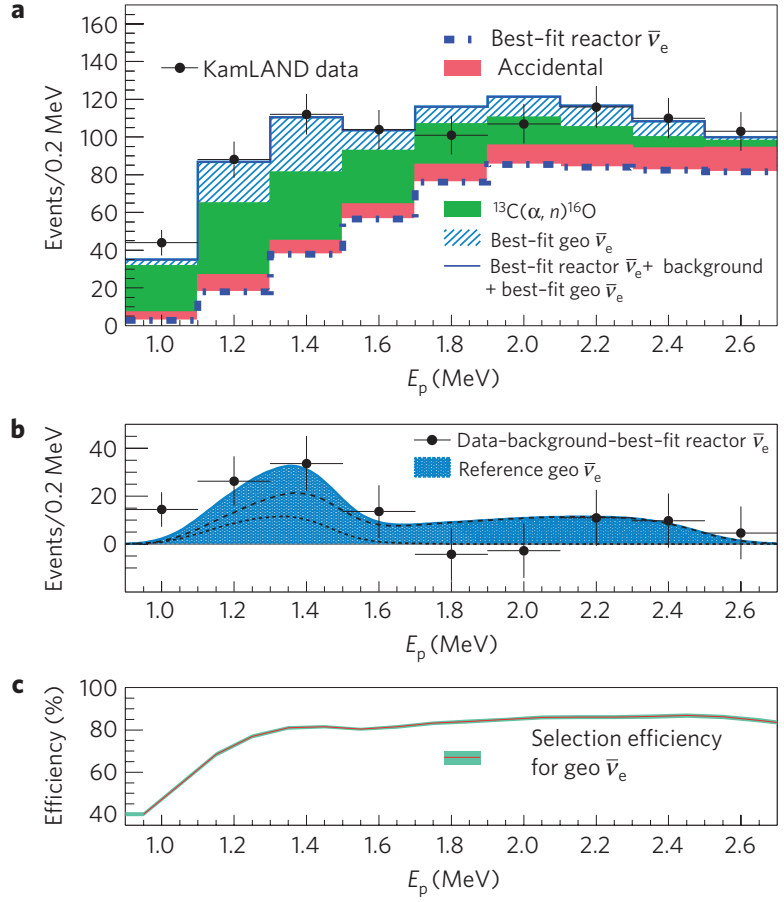

Figure 1 | Prompt energy spectrum and event selection efficiency. a, Prompt energy spectrum of low-energy $\bar{v}_{\mathrm{e}} \mathrm{s}$ in KamLAND. The histograms indicate the backgrounds, whereas the best fit (including geoneutrinos) is shown in blue. b, Background-subtracted energy spectrum. The blue shaded spectrum is the expectation from the reference model, consisting of contributions from $\mathrm{U}$ (dashed curve) and Th (dotted curve). c, Energy dependence of the geoneutrino event selection efficiency averaged over the data-taking period. Statistical uncertainties are shown for the data in $\mathbf{a}$, and uncertainties on the background estimation are added in $\mathbf{b}$.

Taking the neutrino oscillation parameter values $\Delta m_{21}^{2}=$ $7.50_{-0.20}^{+0.19} \times 10^{-5} \mathrm{eV}^{2}$ and $\sin ^{2} 2 \theta_{12}=0.84 \pm 0.03$ from the fit to the data discussed below, the expected number of reactor $\bar{\nu}_{\mathrm{e}}$ events in the geoneutrino energy region (defined as $0.9 \mathrm{MeV}<E_{\mathrm{p}}<$ $2.6 \mathrm{MeV}$ ) is $484.7 \pm 26.5$, including a small contribution from the $\beta$-decay of the long-lived fission products ${ }^{90} \mathrm{Sr},{ }^{106} \mathrm{Ru}$ and ${ }^{144} \mathrm{Ce}$ in spent reactor fuel ${ }^{17}$. Other backgrounds for $\bar{\nu}_{\mathrm{e}}$ detection are mostly from the ${ }^{13} \mathrm{C}(\alpha, n){ }^{16} \mathrm{O}$ reaction in the LS. Including the smaller contributions from accidental coincidences, cosmic-raymuon-induced radioactive isotopes, fast neutrons and atmospheric neutrinos, the total number of events between $0.9 \mathrm{MeV}$ and $2.6 \mathrm{MeV}$ is estimated to be $244.7 \pm 18.4$ (Supplementary Note S2).

We observe 841 candidate $\bar{\nu}_{\mathrm{e}}$ events between $0.9 \mathrm{MeV}$ and $2.6 \mathrm{MeV}$, whereas the predicted number of reactor $\bar{v}_{\mathrm{e}}$ events and other backgrounds is $729.4 \pm 32.3$. Taking the excess as the geoneutrino signal, we obtain $111_{-43}^{+45}$, that is, event yield analysis without energy and time information. The statistical significance is $99.55 \%$.

Figure la shows the fit from a more powerful unbinned maximum-likelihood analysis, which takes into account the event rate, energy and time information in the energy range $0.9 \mathrm{MeV}<$ $E_{\mathrm{p}}<8.5 \mathrm{MeV}$, and simultaneously fits geoneutrinos and reactor $\bar{\nu}_{\mathrm{e}} \mathrm{s}$ including the effect of neutrino oscillations. The oscillation parameters are constrained by solar neutrino flux experiments ${ }^{18}$, including the most recent measurement by Sudbury Neutrino Observatory (SNO; ref. 19). The time of each event gives extra discriminating power because the reactor $\bar{\nu}_{\mathrm{e}}$ background varies with time, as shown in Fig. $2 \mathrm{a}$, as do the accidental and ${ }^{13} \mathrm{C}(\alpha, n){ }^{16} \mathrm{O}$ backgrounds, whereas the geoneutrino rate is constant. As the backgrounds vary, the event rate demonstrates a consistent excess attributable to 

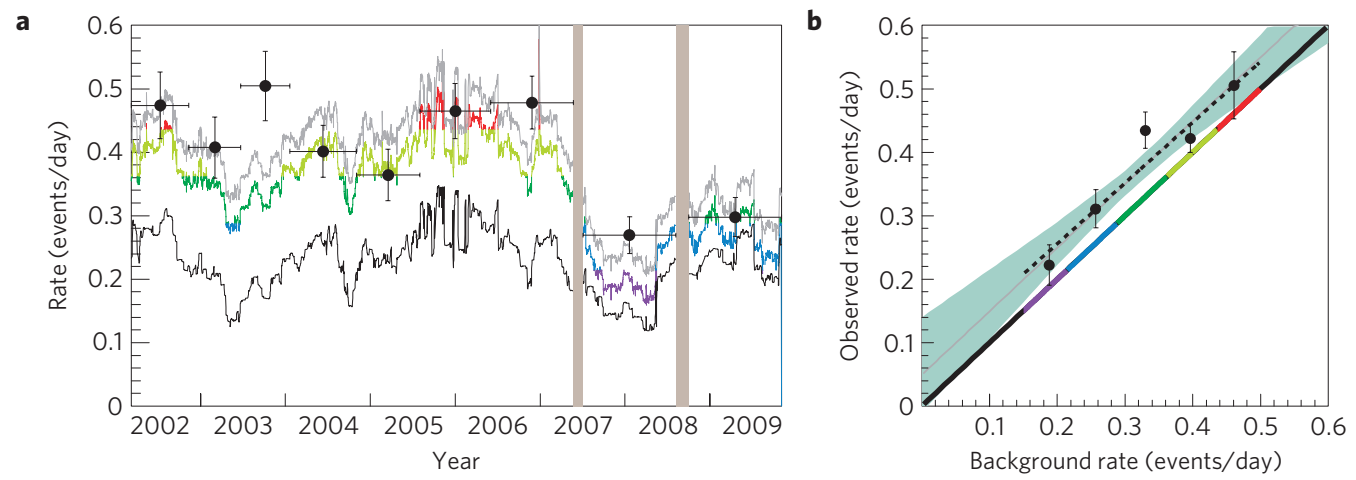

Figure 2 | Event-rate correlation. a, Expected and measured rates at KamLAND for $\bar{\nu}_{\mathrm{e}} \mathrm{s}$ with energies between $0.9 \mathrm{MeV}$ and $2.6 \mathrm{MeV}$. The points indicate the measured rates, whereas the curves show the expected rates for reactor $\bar{v}_{\mathrm{e}} \mathrm{s}$, reactor $\bar{v}_{\mathrm{e}} \mathrm{s}+$ other backgrounds, and reactor $\bar{\nu}_{\mathrm{e}} \mathrm{s}+$ backgrounds + geoneutrinos. The vertical bands correspond to data periods not used owing to high noise resulting from purification activities. $\mathbf{b}$, Measured $\bar{v}_{\mathrm{e}}$ event rates plotted against the expected rate from reactor $\bar{\nu}_{\mathrm{e}} \mathrm{s}+$ other backgrounds. The dotted line is the best linear fit. The shaded region is the $\pm 1 \sigma$ fit envelope. The error bars are statistical only.

geoneutrinos, as clarified by Fig. 2b. The best fit gives 65 and 33 geoneutrino events from ${ }^{238} \mathrm{U}$ and ${ }^{232} \mathrm{Th}$, respectively, when the concentrations of the two isotopes are varied independently. The confidence intervals are shown in Fig. 3a. The result is consistent with the geological reference model ${ }^{4}$ and provides best-fit values for the oscillation parameters of $\Delta m_{21}^{2}=7.50_{-0.20}^{+0.19} \times 10^{-5} \mathrm{eV}^{2}$ and $\sin ^{2} 2 \theta_{12}=0.84 \pm 0.03$, as noted above. These results are slightly more precise than previous measurements ${ }^{2,19}$. Fixing the Earth's Th:U ratio at 3.9, as predicted by the BSE model of ref. 5 from the abundances observed in chondritic meteorites, the total number of geoneutrino events is $106_{-28}^{+29}$, as shown in Fig. 3b. This corresponds to an (oscillated) electron antineutrino flux of $4.3_{-1.1}^{+1.2} \times 10^{6} \mathrm{~cm}^{-2} \mathrm{~s}^{-1}$ from ${ }^{238} \mathrm{U}$ and ${ }^{232} \mathrm{Th}$ at the Earth's surface, whereas the total active geoneutrino flux including all flavours is $7.4_{-1.9}^{+2.1} \times 10^{6} \mathrm{~cm}^{-2} \mathrm{~s}^{-1}$. The uncertainties of the Th:U ratio and oscillation parameters have a negligible effect on the measured geoneutrino flux. The null hypothesis is disfavoured at the $99.997 \%$ confidence level (CL) from assessing the $\Delta \chi^{2}$-profile (Fig. 3b).

Finally, the suggestion that there may exist a natural nuclear reactor in the Earth's core producing $\bar{v}_{\mathrm{e}} \mathrm{s}$ (ref. 20) was tested by adding to the fit a reactor spectrum with a varying amplitude. The spectrum from the hypothetical natural reactor is different from that of power reactors because there is no distortion due to neutrino oscillations over the long path from the Earth's core, only an energy-independent flux suppression. The flux from the natural reactor is assumed constant over the duration of the measurement. In this analysis, solar-neutrino data are used to constrain the neutrino oscillation parameters, and the ${ }^{238} \mathrm{U}$ and ${ }^{232} \mathrm{Th}$ geoneutrino rates are allowed to vary. An upper limit of 5.2 TW at the $90 \%$ $\mathrm{CL}$ was obtained assuming a fission ratio ${ }^{235} \mathrm{U}:{ }^{238} \mathrm{U} \simeq 0.75: 0.25$ (ref. 21), slightly more stringent than a previous KamLAND result ${ }^{2}$. Borexino, being much farther from man-made reactors, provides a significantly lower upper limit of $3 \mathrm{TW}$ at the 95\% CL (ref. 3).

\section{Radiogenic heat estimation and outlook}

Radiogenic contributions to the heating of the Earth in the framework of the BSE model can be determined from the present flux measurement. Variations in the compositional model can be parameterized by weighting factors that multiply the quantities $a_{i}\left(\mathbf{r}^{\prime}\right)$ in equation (1) differently for each region ${ }^{4}$. The mantle is of particular interest because radioactivity is suspected to contribute significantly to mantle convection, which drives plate tectonics and geophysical activity. With existing geochemical and geophysical evidence, there is no consensus on whether two-layer convection, whole-volume convection or something in between is the appropriate description of the mantle. We attempt to focus

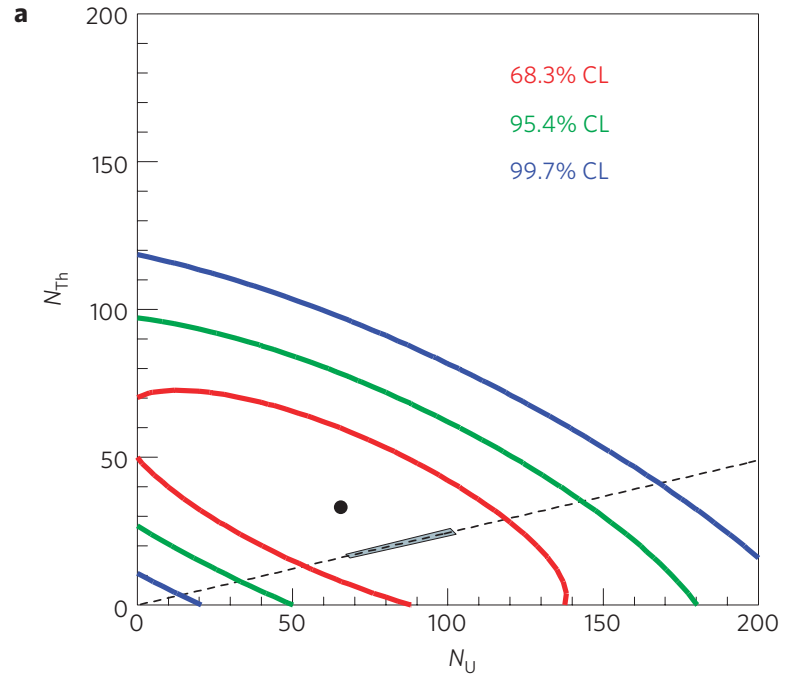

b

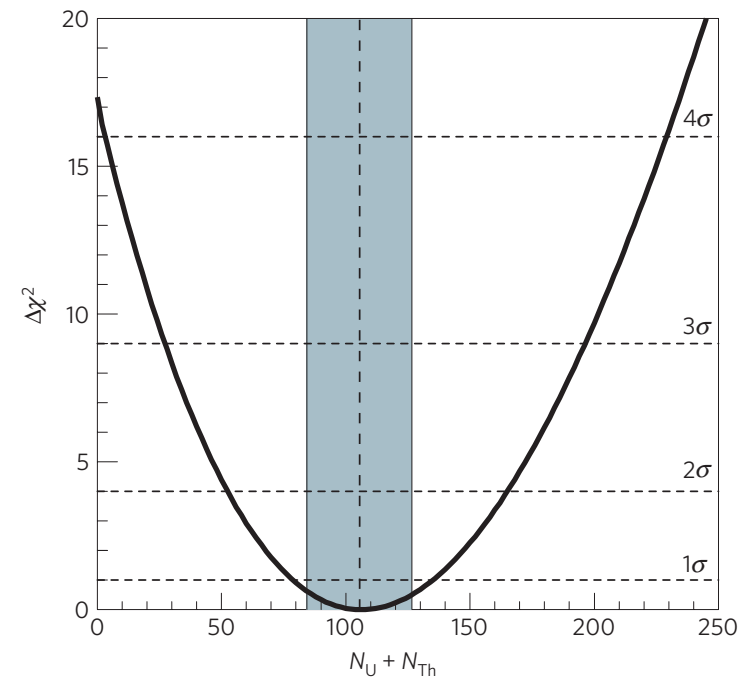

Figure 3 | CL of geoneutrino events. a, CL contours and best-fit point for the observed geoneutrino event rates. The small shaded region is favoured by the reference model ${ }^{4}$. The dashed line is the locus of points expected from the BSE model of ref. 5, Th: $U=3.9 . \mathbf{b}, \Delta \chi^{2}$-profile from the fit to the total number of geoneutrino events discussed in the text. In this case the Th: $U$ ratio is fixed at 3.9. The BSE model prediction is represented by the shaded band ${ }^{5}$. 

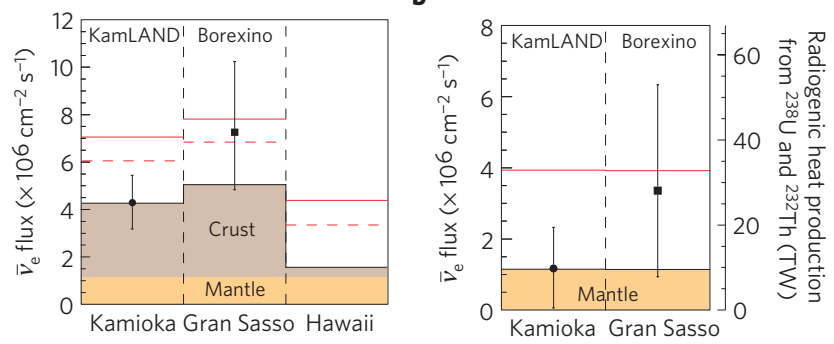

Figure 4 | Measured geoneutrino flux and models. a, Measured geoneutrino flux at Kamioka and Gran Sasso, and expected fluxes at these sites and Hawaii ${ }^{4}$. The solid and dashed red lines represent, respectively, the fluxes for a fully radiogenic model assuming the homogeneous and sunken-layer hypotheses. b, Measured geoneutrino flux after subtracting the estimated crustal contribution. No modelling uncertainties are shown. The right axis shows the corresponding radiogenic heat production assuming a homogeneous mantle. The solid red line indicates the fully radiogenic model where the contributions from the crust (7.0 TW) and the other isotopes $^{6,24}$ (4.3 TW) are subtracted from the total heat flow ${ }^{7}$ (44.2 TW). Error bars, see text.

on the mantle by making simple but appropriate assumptions to constrain the model.

We take the Th:U ratio for each contributing layer to be fixed at the standard BSE model value of 3.9 (ref. 5). The composition of the crust is derived from a BSE model that incorporates the crust and a detailed description of the local geology ${ }^{4}$. As a simplifying hypothesis, $\mathrm{U}$ and $\mathrm{Th}$ are assumed to be uniformly distributed in the mantle. Figure 4a shows the measured geoneutrino fluxes at the Kamioka and Gran Sasso experimental sites along with the predictions for these locations and Hawaii, as an example of an oceanic site with a significantly smaller crustal contribution. Combining the ${ }^{238} \mathrm{U}$ and ${ }^{232} \mathrm{Th}$ geoneutrino measurements of Borexino ${ }^{3}$ and KamLAND we obtain $20.0_{-8.6}^{+8.8} \mathrm{TW}$. The result is in good agreement with the BSE model prediction of $16 \mathrm{TW}$ (ref. 5), as illustrated in Fig. 4b, where the crust contribution is subtracted for clarity.

The fraction of the global heat production from radioactive decay is called the 'Urey ratio'. The mantle contribution alone is referred to as the 'convective Urey ratio'22. Most models, including the BSE model used here, set the convective Urey ratio to about 0.3 , allowing for a substantial fraction of the heat to be of primordial origin. Other models require convective Urey ratios up to $\sim 1.0$ (see discussion in ref. 23). Assuming extra mantle heat contributions of 3.0 TW from other isotope decays ${ }^{6,24}$, the convective Urey ratio deduced from the KamLAND and Borexino data is between 0.18 and 0.67 at the $68 \% \mathrm{CL}$, consistent with 0.3 from the BSE model.

A fully radiogenic model (Urey ratio of 1 ) is constructed by introducing $\mathrm{U}$ and $\mathrm{Th}$ uniformly in the mantle (homogeneous hypothesis) or, alternatively, by putting all of the $\mathrm{U}$ and Th at the mantle-core interface (sunken-layer hypothesis). The latter assumption is used in an attempt to test the compatibility of a fully radiogenic model with the observed geoneutrino flux, by distributing the source as far from the detectors as possible. The fully radiogenic, homogeneous hypothesis is disfavoured at the 97.2\% CL with the combination of KamLAND and Borexino data or at the $98.1 \%$ CL by KamLAND alone. Even within the sunkenlayer hypothesis, the fully radiogenic model is still disfavoured at the $87 \%$ CL using KamLAND data alone.

The radiogenic heat estimation from the geoneutrino flux depends on the modelling of the geology. We account for crustal uncertainties by assuming $17 \%$ and $10 \%$ errors for the $U$ and Th content, including correlated errors as suggested in ref. 9. We use the crustal model of ref. 25, assuming independent errors for each layer (upper, middle and lower crust), and include extra contributions from the error in the mass distribution and the fractional uncertainty in the Th: $\mathrm{U}$ ratiog. The radiogenic heat contribution from ${ }^{238} \mathrm{U}$ and ${ }^{232} \mathrm{Th}$ is estimated to be $19.9_{-9.1}^{+9.2} \mathrm{TW}$ by KamLAND and Borexino data, excluding the fully radiogenic model at the $96.6 \%$ CL. If we use the more recently determined heat-loss rate of $46 \pm 3 \mathrm{TW}$ (ref. 26) the fully radiogenic exclusion increases to $98.0 \% \mathrm{CL}$, slightly enhanced owing to the larger mean value of the heat flow as compared with ref. 7, despite its larger error. We conclude that these uncertainties have little impact on the results at this stage.

It is expected that geoneutrino detectors operated at different locations will significantly improve our knowledge of radiogenic sources in the Earth. Larger detectors distant from commercial reactors will reduce the uncertainties on the measured geoneutrino flux. The geoneutrino flux strongly depends on the distance from thick continental crusts, so the exposure to $\bar{v}_{\mathrm{e}} \mathrm{s}$ at different locations will provide better knowledge of the crustal contribution and greater insight into the mantle. A detector in an oceanic location with small crustal contribution would be very interesting in this regard. The present detectors are all insensitive to ${ }^{40} \mathrm{~K}$, and this will remain an uncertainty unless new geoneutrino detectors with lower threshold are developed.

\section{Methods}

The KamLAND inner detector consists of $1 \mathrm{kt}$ of ultrapure LS contained within a 13-m-diameter spherical balloon made of $135-\mu$ m-thick transparent nylon/EVOH (ethylene vinyl alcohol copolymer) composite film. The balloon is suspended in a bath of purified non-scintillating mineral oil contained inside an 18 -m-diameter stainless-steel sphere. The LS contains $80 \%$ dodecane and $20 \%$ pseudocumene (1,2,4-trimethylbenzene) by volume, as well as $1.36 \pm 0.03 \mathrm{gl}^{-1}$ PPO (2,5-diphenyloxazole) as a fluorophore. The inner surface of the containment sphere is covered by an array of 1,325 specially developed fast 20-inch-diameter photomultiplier tubes (PMTs) masked to 17 inch diameter, and 554 older unmasked 20 inch PMTs. The PMTs provide $34 \%$ solid-angle coverage in total. The containment sphere is surrounded by a $3.2 \mathrm{kt}$ cylindrical water-Cherenkov outer detector instrumented with 225 PMTs of 20 inch diameter. The outer detector acts as a veto counter for muons and helps shield the inner detector from $\gamma$-rays and neutrons produced in the surrounding rock.

Radioactive sources are periodically deployed inside the detector to calibrate its energy response and position-reconstruction accuracy. The reconstruction of event location is important to establish the prompt-delayed event correlation and to define the fiducial volume used in the measurement. After accounting for systematic effects, we find that the deviation of reconstructed event locations from the actual locations is less than $3 \mathrm{~cm}$, from which we derive a $1.8 \%$ uncertainty in the absolute size of the fiducial volume. Source calibration data for the entire fiducial volume are available only for the data recorded before the start of the LS purification campaign in 2007. For the remaining data we carried out calibrations along the vertical axis only. These calibrations were augmented with a study of muon-induced ${ }^{12} \mathrm{~B} /{ }^{12} \mathrm{~N}$ decays ${ }^{27}$, resulting in a larger uncertainly of $2.5 \%$ on the absolute size of the fiducial volume for the post-purification data.

KamLAND was designed and sited primarily to study the phenomenon of neutrino oscillations using reactor $\bar{\nu}_{\mathrm{e}} \mathrm{s}$. Therefore, such $\bar{\nu}_{\mathrm{e}} \mathrm{s}$ represent the largest background in the present measurement because their energy spectrum partially overlaps that of geoneutrinos. Substantial discrimination between the two is achieved not only by fitting their energy spectra but also by exploiting the fact that the reactor $\bar{v}_{\mathrm{e}}$ rate varies with the output of the power plants whereas the geoneutrino rate can be taken as constant over the timescale of the experiment.

The $\bar{v}_{\mathrm{e}}$ event-selection criteria are optimized as a function of energy to maximize the sensitivity to geoneutrinos while rejecting the accidental background from radioactive contaminants in the detector. The event selection is based on the discriminant $L=f_{\bar{v}_{\mathrm{e}}} /\left(f_{\bar{v}_{\mathrm{e}}}+f_{\text {acc }}\right)$, where $f_{\bar{v}_{\mathrm{e}}}$ and $f_{\text {acc }}$ are probability density functions for $\bar{\nu}_{\mathrm{e}}$ signals and accidental backgrounds, respectively. These probability density functions are based on six parameters $\left(E_{\mathrm{p}}, E_{\mathrm{d}}, \Delta R, \Delta T, R_{\mathrm{p}}, R_{\mathrm{d}}\right)$, which represent, respectively, the prompt and delayed event energies, their relative separations in space and time and their radial distances from the detector centre. Owing to an observed variation of the background rate with time, the probability density function for accidental backgrounds is a time-dependent function constructed by dividing the data set into five time periods. For the discrimination of accidental backgrounds, we determine a selection value, $L_{\mathrm{cut}}\left(E_{\mathrm{p}}\right)$, to maximize the figure of merit $S / \sqrt{S+B_{\text {acc }}}$ for each prompt energy interval of $0.1 \mathrm{MeV}$, where $S$ denotes the expected signal rate and $B_{\text {acc }}$ corresponds to the accidental background rate. The selection efficiency and its uncertainty are obtained by comparing Monte Carlo simulations with ${ }^{68} \mathrm{Ge}$ and ${ }^{241} \mathrm{Am}^{9} \mathrm{Be}$ source calibration data. The selection efficiencies for geoneutrino signals produced by $\mathrm{U}$ and $\mathrm{Th}$ decays with energies 
above threshold are $80.7 \%$ and $75.1 \%$, respectively, where the difference arises from their dissimilar energy spectra. The total systematic errors for both numbers are $2.6 \%$ for the pre-purification data set and $3.4 \%$ for the post-purification.

\section{Received 5 November 2010; accepted 14 June 2011;} published online 17 July 2011

\section{References}

1. Araki, T. et al. Experimental investigation of geologically produced antineutrinos with KamLAND. Nature 436, 499-503 (2005).

2. Abe, S. et al. Precision measurement of neutrino oscillation parameters with KamLAND. Phys. Rev. Lett. 100, 221803 (2008).

3. Bellini, G. et al. Observation of geo-neutrinos. Phys. Lett. B 687, 299-304 (2010).

4. Enomoto, S., Ohtani, E., Inoue, K. \& Suzuki, A. Neutrino geophysics with KamLAND and future prospects. Earth Planet. Sci. Lett. 258, 147-159 (2007).

5. McDonough, W. F. \& Sun, S-s. The composition of the Earth. Chem. Geol. 120, 223-253 (1995).

6. Arevalo, R. Jr, McDonough, W. F. \& Luong, M. The K/U ratio of the silicate Earth: Insights into mantle composition, structure and thermal evolution. Earth Planet. Sci. Lett. 278, 361-369 (2009).

7. Pollack, H. N., Hurter, S. J. \& Johnson, J. R. Heat flow from the Earth's interior: Analysis of the global data set. Rev. Geophys. 31, 267-280 (1993).

8. Fiorentini, G., Lissia, M. \& Mantovani, F. Geo-neutrinos and earth's interior. Phys. Rep. 453, 117-172 (2007).

9. Fogli, G. L., Lisi, E., Palazzo, A. \& Rotunno, A. M. Geo-neutrinos: A systematic approach to uncertainties and correlations. Earth Moon Planets 99, 111-130 (2006).

10. Giunti, C. \& Kim, C. W. Fundamentals of Neutrino Physics and Astrophysics (Oxford Univ. Press, 2007).

11. Abe, S. et al. Production of radioactive isotopes through cosmic muon spallation in KamLAND. Phys. Rev. C 81, 025807 (2010).

12. Vogel, P. \& Beacom, J. F. Angular distribution of neutron inverse beta decay, $\bar{v}_{\mathrm{e}}+p \rightarrow e^{+}+n$. Phys. Rev. D 60, 053003 (1999).

13. Schreckenbach, K., Colvin, G., Gelletly, W. \& Von Feilitzsch, F. Determination of the antineutrino spectrum from ${ }^{235} \mathrm{U}$ thermal neutron fission products up to $9.5 \mathrm{MeV}$. Phys. Lett. B 160, 325-330 (1985).

14. Hahn, A. A. et al. Antineutrino spectra from ${ }^{241} \mathrm{Pu}$ and ${ }^{239} \mathrm{Pu}$ thermal neutron fission products. Phys. Lett. B 218, 365-368 (1989).

15. Vogel, P. Reactor antineutrino spectra and their application to antineutrino-induced reactions. II. Phys. Rev. C 24, 1543-1553 (1981).

16. Achkar, B. et al. Comparison of anti-neutrino reactor spectrum models with the Bugey 3 measurements. Phys. Lett. B 374, 243-248 (1996).

17. Kopeikin, V. I., Mikaelyan, L. A. \& Sinev, V. V. Inverse beta decay in a nonequilibrium antineutrino flux from a nuclear reactor. Phys. Atomic Nucl. 64, 849-854 (2001).

18. Bahcall, J. N., Serenelli, A. M. \& Basu, S. New solar opacities, abundances, helioseismology, and neutrino fluxes. Astrophys. J. 621, 85-88 (2005).
19. Aharmim, B. et al. Low-energy-threshold analysis of the Phase I and Phase II data sets of the Sudbury Neutrino Observatory. Phys. Rev. C 81, 055504 (2010).

20. Herndon, J. M. Nuclear georeactor origin of oceanic basalt ${ }^{3} \mathrm{He} /{ }^{4} \mathrm{He}$, evidence, and implications. Proc. Natl Acad. Sci. USA 100, 3047-3050 (2003).

21. Herndon, J. M. \& Edgerley, D. A. Background for terrestrial antineutrino investigations: Radionuclide distribution, georeactor fission events, and boundary conditions on fission power production. Preprint at http://arxiv.org/abs/hep-ph/0501216v4 (2005).

22. Korenaga, J. Urey ratio and the structure and evolution of Earth's mantle. Rev. Geophys. 46, RG2007 (2008).

23. Lyubetskaya, T. \& Korenaga, J. Chemical composition of Earth's primitive mantle and its variance: 2 . Implications for global geodynamics. J. Geophys. Res. 112, B03212 (2007).

24. Enomoto, S. Experimental study of geoneutrinos with KamLAND. Earth Moon Planets 99, 131-146 (2006).

25. Rudnick, R. L., Gao, S., Holland, H. D. \& Turekian, K. K. Treatise on Geochemistry: Composition of the Continental Crust, Vol. 3 1-64 (Pergamon, 2003).

26. Jaupart, C., Labrosse, S., Mareschal, J. C. \& Schubert, G. Treatise on Geophysics: Temperatures, Heat and Energy in the Mantle of the Earth Vol. 7 253-303 (Elsevier, 2007).

27. Berger, B. E. et al. The KamLAND full-volume calibration system. J. Instrum. 4, P04017 (2009).

\section{Acknowledgements}

We thank E. Ohtani and W. F. McDonough for advice and guidance. The KamLAND experiment is supported by a Grant-in-Aid for Specially Promoted Research under grant 16002002 of the Japanese Ministry of Education, Culture, Sports, Science and Technology; the World Premier International Research Center Initiative (WPI Initiative), MEXT, Japan; and the US Department of Energy (DOE) grants DEFG03-00ER41138 and DE-AC02-05CH11231, as well as other DOE grants to individual institutions. The reactor data are provided by courtesy of the following electric associations in Japan: Hokkaido, Tohoku, Tokyo, Hokuriku, Chubu, Kansai, Chugoku, Shikoku and Kyushu Electric Power Companies, Japan Atomic Power Company and Japan Atomic Energy Agency. The Kamioka Mining and Smelting Company has provided service for activities in the mine.

\section{Author contributions}

All authors contributed equally to the work presented in this study.

\section{Additional information}

The authors declare no competing financial interests. Supplementary information accompanies this paper on www.nature.com/naturegeoscience. Reprints and permissions information is available online at http://www.nature.com/reprints. Correspondence and requests for materials should be addressed to I.S.

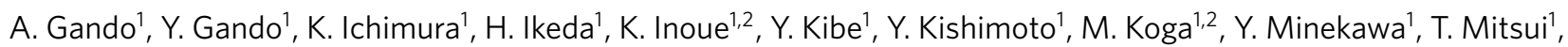

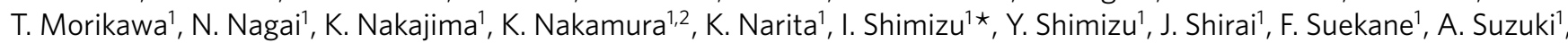
H. Takahashi', N. Takahashi', Y. Takemoto', K. Tamae', H. Watanabe', B. D. Xu' , H. Yabumoto', H. Yoshida', S. Yoshida', S. Enomoto ${ }^{2}$, A. Kozlov², H. Murayama2,3, C. Grant ${ }^{4}$, G. Keefer ${ }^{4}$, A. Piepke2,4 T. I. Banks' ${ }^{3}$, T. Bloxham³ ${ }^{3}$, J. A. Detwiler ${ }^{3}$,

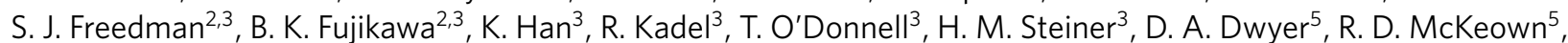

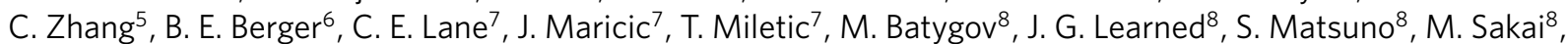
G. A. Horton-Smith ${ }^{2,9}$, K. E. Downum ${ }^{10}$, G. Gratta ${ }^{10}$, K. Tolich ${ }^{10}$, Y. Efremenko ${ }^{2,11}$, O. Perevozchikov ${ }^{11}$, H. J. Karwowski ${ }^{12,13}$, D. M. Markoff ${ }^{12,13}$, W. Tornow ${ }^{12,13}$, K. M. Heeger ${ }^{2,14}$ and M. P. Decowski ${ }^{2,15}$

${ }^{1}$ Research Center for Neutrino Science, Tohoku University, Sendai 980-8578, Japan, ${ }^{2}$ Institute for the Physics and Mathematics of the Universe, Tokyo University, Kashiwa 277-8568, Japan, ${ }^{3}$ Physics Department, University of California, Berkeley and Lawrence Berkeley National Laboratory, Berkeley, California 94720, USA, ${ }^{4}$ Department of Physics and Astronomy, University of Alabama, Tuscaloosa, Alabama 35487, USA, ${ }^{5}$ W. K. Kellogg Radiation Laboratory, California Institute of Technology, Pasadena, California 91125, USA, ${ }^{6}$ Department of Physics, Colorado State University, Fort Collins, Colorado 80523, USA, ${ }^{7}$ Physics Department, Drexel University, Philadelphia, Pennsylvania 19104, USA, ${ }^{8}$ Department of Physics and Astronomy, University of Hawaii at Manoa, Honolulu, Hawaii 96822, USA, ${ }^{9}$ Department of Physics, Kansas State University, Manhattan, Kansas 66506, USA, ${ }^{10}$ Physics Department, Stanford University, Stanford, California 94305, USA, ${ }^{11}$ Department of Physics and Astronomy, University of Tennessee, Knoxville, Tennessee 37996, USA, ${ }^{12}$ Triangle Universities Nuclear Laboratory, Durham, North Carolina 27708, USA, ${ }^{13}$ Physics Departments at Duke University, North Carolina Central University, and the University of North Carolina at Chapel Hill, USA, ${ }^{14}$ Department of Physics, University of Wisconsin, Madison, Wisconsin 53706 , USA, ${ }^{15}$ Nikhef, Science Park 105, 1098 XG Amsterdam, The Netherlands. *e-mail: shimizu@awa.tohoku.ac.jp. 